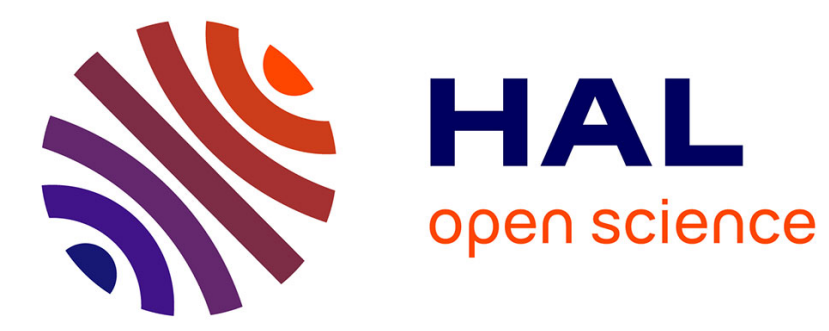

\title{
Captures de Lobesia botrana Den. \& Schiff. (Lepidoptera, Tortricidae) au piège sexuel en relation avec la dose en phéromone du diffuseur
}

Roger Roehrich, Jean-Paul Carles, André Dreuilhe, Francis Vonderheyden

\section{- To cite this version:}

Roger Roehrich, Jean-Paul Carles, André Dreuilhe, Francis Vonderheyden. Captures de Lobesia botrana Den. \& Schiff. (Lepidoptera, Tortricidae) au piège sexuel en relation avec la dose en phéromone du diffuseur. Agronomie, 1983, 3 (9), pp.925-930. hal-00884591

\section{HAL Id: hal-00884591 https://hal.science/hal-00884591}

Submitted on 1 Jan 1983

HAL is a multi-disciplinary open access archive for the deposit and dissemination of scientific research documents, whether they are published or not. The documents may come from teaching and research institutions in France or abroad, or from public or private research centers.
L'archive ouverte pluridisciplinaire HAL, est destinée au dépôt et à la diffusion de documents scientifiques de niveau recherche, publiés ou non, émanant des établissements d'enseignement et de recherche français ou étrangers, des laboratoires publics ou privés. 


\title{
Captures de Lobesia botrana Den. \& Schiff. (Lepidop- tera, Tortricidae) au piège sexuel en relation avec la dose en phéromone du diffuseur
}

\author{
Roger ROEHRICH, Jean-Paul CARLES, André DREUILHE (*) \& Francis VONDERHEYDEN (*) \\ I.N.R.A., Station de Zoologie, Centre de Recherches de Bordeaux, F 33140 Pont-de-la-Maye \\ $\left(^{*}\right)$ Service de la Protection des Végétaux, Région Aquitaine, F 33150 Cenon-la-Morlette
}

RÉSUMÉ

\begin{abstract}
Plusieurs essais de piégeage sexuel de Lobesia botrana ont été effectués avec différentes doses de phéromone E 7, Z 9 DDA. Le classement d'efficacité a été variable et semble dépendre de plusieurs facteurs. Les fortes doses $(10 \mathrm{mg})$ sont attractives à des distances plus grandes et sont favorisées par la situation des pièges au milieu de grandes surfaces de vigne. Des doses 100 à 1000 fois plus faibles $(1 \mathrm{ou} 10 \mu \mathrm{g})$ que celle utilisée pour l'avertissement améliorent le rendement des captures parmi les papillons attirés et se placent ainsi en tête lorsque les conditions locales sont moins favorables à l'action à grande distance.
\end{abstract}

Mots clés additionnels : Eudémis, avertissement agricole, vignoble.

Several experiments have been performed on sexual trapping of $L$. botrana males with different pheromone concentrations (E 7, Z 9 DDA). Performance classification was difficult and seemed to depend of several factors. High concentrations ( $10 \mathrm{mg}$ in the dispenser) seemed to be attractive at long range and this effect was enhanced by the trap location in the middle of large vineyard areas. Low concentrations (1 to $10 \mu \mathrm{g})$ gave a better yield for trapping local populations and seemed preferable when the situation was unsuitable for longrange attraction.

Additional key words : Vine moth, agricultural forecasting, vineyard.

\section{INTRODUCTION}

Le piégeage sexuel des mâles de lépidoptères est un élément important pour déterminer les dates des interventions (avertissements agricoles). De plus, des essais ont été effectués pour rechercher s'il est possible d'en tirer des données quantitatives permettant d'évaluer l'importance locale du risque (RoEHRICH \& SCHMID, 1979). Enfin, il est probable que l'attraction joue un rôle dans la confusion sexuelle : l'attirance des mâles vers de nombreux points de diffusion disséminés dans la parcelle les empêcheraient de localiser les femelles (ROEHRICH et al., 1979 ; ROTHSCHILD, 1980).

Les essais comparatifs de piégeage sexuel avec des doses variées de phéromones sont nombreux et leurs résultats ont été très différents selon les espèces et les conditions d'expérimentation (STOCKEl \& SuREAU, 1980). Nos propres recherches sur Lobesia botrana Den. et Schiff. nous ayant donné des résultats variables (ROEHRICH et al., 1976, 1977), il nous a paru utile de les rassembler et de les compléter par une expérimentation nouvelle présentant dans 2 vignobles une large gamme de doses aux insectes. Parallèlement, nous avons effectué un autre type d'essais en n'utilisant qu'un seul piège à la fois par parcelle: les résultats feront l'objet d'un autre article.

\section{MATÉRIEL ET MÉTHODES}

Le composé majoritaire de la sécrétion phéromonale émis par la femelle vierge de $L$. botrana est l'acétoxy-1 dodécadiène-7 E, $9 \mathrm{Z}$ (RoElofs et al., 1973 ; DesCoIns et al., 1974). 
Utilisé seul, ce composé induit les différentes séquences comportementales intervenant dans l'attraction du mâle par la femelle (LALANNE CASSOU, 1979). Il a été synthétisé au Laboratoire des Médiateurs chimiques de l'I.N.R.A. selon un procédé original (DESCOINS et al., 1977). La pureté stérique du produit de synthèse est voisine de $97 \mathrm{p} .100$. Il est adsorbé sur un bouchon en caoutchouc, type bouchon pour tube à allergie (Ets LEUNE, Orsay) placé au centre d'un piège englué, modèle phérocon (ZOECON Corporation) de 1974 à 1976, puis I.N.R.A. (STOCKEL, 1976).

Les pièges étaient disposés dans les parcelles, éloignés de $15 \mathrm{~m}$ environ l'ın de l'autre. Le dispositif était, soit un essai bloc avec 3 répétitions, soit un essai en rotation dans lequel chaque piège est déplacé régulièrement, occupant successivement dans la parcelle toutes les implantations afin d'éviter les distortions dues à des situations éventuellement plus favorables aux captures.

Les pièges, en 1974 et 1975 , faisaient partie de dispositifs complexes de rotation destinés à comparer des mélanges d'isomères de phéromones avec des adjuvants, des doses et des diffuseurs, d'où nous avons extrait ce qui concernait seulement les doses de E 7, Z 9 DDA.

En 1976, nous avons comparé en essai bloc des doses très faibles $(0,1$ et $1 \mu \mathrm{g})$ dans le but de rechercher si l'attractivité d'un autre isomère : le $\mathrm{E} 7, \mathrm{E} 9 \mathrm{DDA}$ pouvait tenir ou non à la présence de traces de l'isomère $\mathrm{E} 7, \mathrm{Z} 9 \mathrm{DDA}$.

En 1977, ayant observé, lors d'une expérience de confusion en petites parcelles, une activité des mâles autour de diffuseurs chargés à $12 \mathrm{mg}$ de phéromone (ROEHRICH et al., 1979), nous avons comparé, en essai bloc, des doses élevées $(1,10$ et $100 \mathrm{mg})$ à notre référence $(100 \mu \mathrm{g})$.

Ayant eu jusque-là des résultats difficilement interprétables, nous avons repris, en 1982, dans un même dispositif l'ensemble des doses entre $1 \mu \mathrm{g}$ et $10 \mathrm{mg}$ : dans chacun des 2 vignobles (Chàteau Baret à Villenave d'Ornon et vignoble de M. Boursaud à Artigues), nous avons disposé 5 pièges en rotation pendant toute la période de présence des papillons (avril à septembre). Les analyses statistiques utilisent comme répétitions les relevés de piégeage (tous les jours ou 3 fois par semaine). Les tests non paramétriques utilisés ont été : le test de WILCOXSON lorsqu'il n'y avait que 2 doses en comparaison et le test de FRIEDMAN dans le cas contraire. Lorsque ce test indiquait l'existence de différences significatives nous avons, au moyen du test de KRAMER, réparti les résultats en 3 groupes bien caractérisés.

\section{RESULTATS}

On trouvera au tableau 1 un résumé des essais de 1974 à 1977 en valeur réelle (nombre de papillons capturés pendant la durée de l'essai) et en pourcentage du total obtenu avec $100 \mu \mathrm{g}$ pour faciliter les comparaisons.

Il apparaît une hétérogénéité importante, mais on peut remarquer également que les différences significatives et importantes entre les doses s'étageant de $10 \mu \mathrm{g}$ à $100 \mathrm{mg}$ (soit 10000 fois plus) sont rares et pas toujours dans le même sens. Ainsi, en 1974 , la dose de $12,5 \mu \mathrm{g}$ a capturé beaucoup plus de mâles que la dose de $125 \mu \mathrm{g}$, alors qu'en 1975 dans le même vignoble, la situation est inversée.

Dans l'essai en 2 sites de 1982, pendant toute la période d'activité des papillons, nous avons cherché à individualiser les différentes séquences. Au Château Baret, les 3 vols habituels pour la région étaient bien séparés par des périodes avec très peu de captures et il a été facile de les caractériser. A Artigues au contraire, la pointe du $1^{\text {er }}$ vol est beaucoup plus tardive et les captures sont continues; aussi nous avons dû arbitrairement diviser l'année en 3 périodes centrées chacune sur les vols observés au Château Baret (tabl. 2).

TABLEAU 1

Influence de la dose sur le piégeage: Essais de 1974 à 1977. En italiques, données ramenées à 100 pour $100 \mu \mathrm{g}$. Action of concentration on trapping : experiments from 1974 to 1977. In italics, percentage of the number of males trapped by $100 \mu \mathrm{g}$ dispensers.

\begin{tabular}{|c|c|c|c|c|c|c|c|c|c|}
\hline Dose & $0,1 \mu \mathrm{g}$ & $0,5 \mu \mathrm{g}$ & $1 \mu \mathrm{g}$ & $10 \mu \mathrm{g}$ & $100 \mu \mathrm{g}$ & $1 \mathrm{mg}$ & $10 \mathrm{mg}$ & $100 \mathrm{mg}$ & \\
\hline \multirow[t]{2}{*}{$\begin{array}{c}1974 \\
\text { (rotation) }\end{array}$} & & & & & $\begin{array}{l}144 \\
100\end{array}$ & $\begin{array}{l}178 \\
123\end{array}$ & & & NS \\
\hline & & & & $\begin{array}{l}401 \\
400\end{array}$ & $\begin{array}{r}99 \\
100\end{array}$ & & & & $\left(^{*}\right)$ \\
\hline \multirow[t]{4}{*}{$\begin{array}{c}1975 \\
\text { (rotation) }\end{array}$} & & & & $\begin{array}{l}91 \\
42\end{array}$ & $\begin{array}{l}216 \\
100\end{array}$ & & & & $\left(^{*}\right)$ \\
\hline & & & 91 & 74 & & & & & NS \\
\hline & & & & $\begin{array}{r}140 \\
71\end{array}$ & $\begin{array}{l}193 \\
100\end{array}$ & & & & NS \\
\hline & & & $\begin{array}{l}61 \\
99\end{array}$ & $\begin{array}{l}42 \\
67\end{array}$ & $\begin{array}{r}62 \\
100\end{array}$ & & & & NS \\
\hline \multirow[t]{2}{*}{$\begin{array}{c}1976 \\
\text { (répétitions) }\end{array}$} & $\begin{array}{l}7 \\
5\end{array}$ & $\begin{array}{l}24 \\
25\end{array}$ & & & $\begin{array}{l}141 \\
100\end{array}$ & & & & $\left(^{*}\right)$ \\
\hline & & & $\begin{array}{r}126 \\
19\end{array}$ & $\begin{array}{r}190 \\
44\end{array}$ & $\begin{array}{l}437 \\
.100\end{array}$ & $\begin{array}{l}467 \\
108\end{array}$ & & & NS \\
\hline $\begin{array}{c}1977 \\
\text { (répétitions) }\end{array}$ & & & & & $\begin{array}{l}482 \\
100\end{array}$ & $\begin{array}{l}795 \\
165\end{array}$ & $\begin{array}{l}658 \\
137\end{array}$ & $\begin{array}{l}489 \\
102\end{array}$ & NS \\
\hline
\end{tabular}


TABLEAU 2

Captures en 1982 dans 2 vignobles avec rotation des pièges. En italiques, classement de rang moyen. Pourcentage du total de la période. Trapping in 1982 in two vineyards with rotation of the traps. En italics, mean rank class. Per cent of the total of the period.

\begin{tabular}{|c|c|c|c|c|c|c|c|}
\hline $\begin{array}{l}\text { Lieu } \\
\text { et date }\end{array}$ & Dose & $1 \mu \mathrm{g}$ & $10 \mu \mathrm{g}$ & $100 \mu \mathrm{g}$ & $1 \mathrm{mg}$ & $10 \mathrm{mg}$ & $\begin{array}{c}\text { Test de } \\
\text { Friedman } \\
\text { X2 }\end{array}$ \\
\hline \multirow[b]{2}{*}{ Château } & $\begin{array}{c}1^{\mathrm{er}} \text { vol } \\
16.4 / 12.5\end{array}$ & $\begin{array}{l}3 \\
2,17 \\
6 \%\end{array}$ & $\begin{array}{c}13 \\
3,07 \\
25 \%\end{array}$ & $\begin{array}{c}12 \\
3,33 \\
23 \%\end{array}$ & $\begin{array}{c}14 \\
3,47 \\
27 \%\end{array}$ & $\begin{array}{c}10 \\
2,97 \\
19 \%\end{array}$ & NS $10-20 \%$ \\
\hline & $\begin{array}{c}2^{\mathrm{e}} \text { vol } \\
18.6 / 9.7\end{array}$ & $\begin{array}{c}16 \\
3,22 \\
22 \%\end{array}$ & $\begin{array}{c}15 \\
3,28 \\
20 \%\end{array}$ & $\begin{array}{c}21 \\
3,19 \\
28 \%\end{array}$ & $\begin{array}{c}12 \\
2,63 \\
16 \%\end{array}$ & $\begin{array}{c}10 \\
2,69 \\
14 \%\end{array}$ & $\begin{array}{l}2,56 \\
\text { NS }\end{array}$ \\
\hline \multirow[t]{3}{*}{ Baret } & $\begin{array}{l}3^{\mathrm{c}} \mathrm{vol} \\
2.8 / 13.9\end{array}$ & $\begin{array}{c}65 \\
3,78 \\
26 \%\end{array}$ & $\begin{array}{c}74 \\
3,15 \\
30 \%\end{array}$ & $\begin{array}{c}35 \\
2,65 \\
14 \%\end{array}$ & $\begin{array}{c}60 \\
3,30 \\
24 \%\end{array}$ & $\begin{array}{l}15 \\
2,11 \\
6 \%\end{array}$ & $\begin{array}{c}15,12 \\
(* *)\end{array}$ \\
\hline & Total & $\begin{array}{l}82 \\
22 \%\end{array}$ & $\begin{array}{l}105 \\
28 \%\end{array}$ & $\begin{array}{l}67 \\
18 \%\end{array}$ & $\begin{array}{l}87 \\
25 \%\end{array}$ & $\begin{array}{l}33 \\
9 \%\end{array}$ & $\begin{array}{l}1,50 \\
\text { NS }\end{array}$ \\
\hline & $\begin{array}{l}1^{\mathrm{Tc}} \text { période } \\
19.4 / 18.6\end{array}$ & $\begin{array}{c}174 \\
2,17 \\
13 \%\end{array}$ & $\begin{array}{c}251 \\
3,11 \\
19 \%\end{array}$ & $\begin{array}{c}208 \\
2,70 \\
15 \%\end{array}$ & $\begin{array}{c}279 \\
2,96 \\
21 \%\end{array}$ & $\begin{array}{r}427 \\
4,0 \\
33 \%\end{array}$ & $\begin{array}{l}17,70 \\
(* *)\end{array}$ \\
\hline \multirow[t]{3}{*}{ Artigues } & $\begin{array}{c}2^{\mathrm{c}} \text { période } \\
19.6 / 26.7\end{array}$ & $\begin{array}{c}74 \\
2,20 \\
11 \%\end{array}$ & $\begin{array}{l}80 \\
2,40 \\
12 \%\end{array}$ & $\begin{array}{c}94 \\
2,23 \\
14 \%\end{array}$ & $\begin{array}{c}196 \\
4,03 \\
29 \%\end{array}$ & $\begin{array}{c}221 \\
4,13 \\
33 \%\end{array}$ & $\begin{array}{l}23,64 \\
(* *)\end{array}$ \\
\hline & $\begin{array}{l}3^{c} \text { période } \\
28.7 / 10.9\end{array}$ & $\begin{array}{c}246 \\
3,17 \\
28 \%\end{array}$ & $\begin{array}{c}205 \\
3,14 \\
23 \%\end{array}$ & $\begin{array}{c}141 \\
2,89 \\
16 \%\end{array}$ & $\begin{array}{c}141 \\
2,75 \\
16 \%\end{array}$ & $\begin{array}{c}156 \\
3,06 \\
17 \%\end{array}$ & 0,90 \\
\hline & Total & $\begin{array}{l}494 \\
17 \%\end{array}$ & $\begin{array}{l}536 \\
19 \%\end{array}$ & $\begin{array}{l}443 \\
15 \%\end{array}$ & $\begin{array}{l}616 \\
21 \%\end{array}$ & $\begin{array}{l}802 \\
28 \%\end{array}$ & $\begin{array}{c}21,38 \\
(* *)\end{array}$ \\
\hline
\end{tabular}

Une $1^{\text {ère }}$ analyse (test de FRIEDMAN) a montré dans les 2 cas une homogénéité des captures selon la position du piège dans le vignoble (probabilité supérieure à 90 p. 100 d'homogénéité pour les 2 premières générations au Château Baret, supérieure à 60 p. 100 dans les autres cas). Nous avons donc pu poursuivre l'étude en prenant comme variable chaque relevé et non la somme des 5 relevés nécessaires pour que chaque piège soit placé dans les 5 positions différentes, ce qui aurait été indispensable en cas d'hétérogénéité.

Appliqué non plus aux positions mais aux doses, le test de FRIEDMAN nous indique des différences significatives au seuil de 1 p. 100 pour le $3^{\mathrm{e}}$ vol du Château Baret et les 2 premières périodes à Artigues, et entre 10 et 20 p. 100 pour le $1^{\text {cr }}$ vol au Château Baret. Dans les 3 premiers cas, nous avons pu montrer, à l'aide du test de KRAMER, au seuil 5 p. 100, les différences suivantes entre l'action des doses:

1. Au $3^{\mathrm{c}}$ vol au Château Baret, la dose de $10 \mathrm{mg}$ attire moins que les autres.

2. Pendant la $1^{\text {̀̀re }}$ période à Artigues, la dose de $10 \mathrm{mg}$ attire plus que les autres.

3. Pendant la $2^{\mathrm{e}}$ période à Artigues, les doses se séparent en 2 groupes très caractérisés : les doses de $1 \mathrm{mg}$ et $10 \mathrm{mg}$ sont nettement plus attractives que les doses de $1 \mu \mathrm{g}, 10 \mu \mathrm{g}$, et $100 \mu \mathrm{g}$.

De plus, lors du $1^{\text {er }}$ vol au Château Baret, la dose de $1 \mu \mathrm{g}$ n'a capturé que 3 papillons et son classement de rang moyen est très faible. Il y a donc une tendance très nette permettant de classer cette dose en dessous des autres.
Si nous considérons l'ensemble de l'année, il n'y a aucune différence significative au Château Baret (les tendances sont de sens contraires entre le $1^{\text {er }}$ et le $3^{\mathrm{e}} \mathrm{vol}$ ), mais à Artigues, au test de KRAMER, la dose $10 \mathrm{mg}$ se montre plus attractive et la dose $100 \mu \mathrm{g}$ moins attractive que l'ensemble des 3 autres.

\section{DISCUSSION}

Les essais de 1982 ont donc confirmé l'hétérogénéité du classement d'efficacité des doses selon les vignobles et les périodes. Quelles hypothèses pouvons-nous faire pour expliquer ces divergences?

L'âge des capsules et par conséquent la quantité réelle d'attractif, la température au moment de l'essai déterminant le taux d'évaporation et la pureté du produit ne semblent pas pouvoir expliquer les différences entre les 2 vignobles, le chargement ayant eu lieu en même temps, à partir du même flacon de E 7, Z 9 DDA et l'expérimentation ayant eu lieu à la même époque avec changement de capsules aux mêmes dates. Il faut cependant noter que dans le vignoble d'Artigues, le cycle de l'eudémis est nettement plus tardif qu'au Château Baret, ce qui pourrait traduire soit une différence notable dans les températures (que nous n'avons pas pu mesurer), soit la présence d'une population d'insectes présentant des différences de réponse aux conditions extérieures. La direction du vent, la position des pièges par rapport à la situation des papillons dans les vignobles pourraient apporter une explication et les travaux de BAKER 
\& ROELOFS (1981) sur un autre Tortricide Grapholitha molesta Busck. permettraient peut-être de comprendre ces différences.

Ces auteurs ont montré que la distance jusqu'à laquelle le signal chimique est perçu et modifie le comportement, augmente avec la dose alors que, au contraire, une plus grande proportion des papillons attirés se pose près du diffuseur lorsque les doses sont faibles. Le vignoble du Château Baret est isolé mais groupe $10 \mathrm{ha}$. La parcelle d'essai est située à l'est du vignoble dans lequel est implanté un autre essai de phéromones utilisant 6 pièges, placés chacun au milieu d'une parcelle de 1 ha environ. Les vents dominants étant de secteur ouest, la zone où la phéromone risque d'être transportée par le déplacement d'air ne comporte pas de vigne au-delà de $100 \mathrm{~m}$ environ : les fortes doses ne peuvent donc pas faire preuve de leur supériorité probable dans la distance d'attraction. Si elles gênent l'arrivée à proximité du diffuseur, on peut comprendre ainsi la meilleure efficacité relative des doses les plus faibles (sauf peut-être pendant le mois d'avril où les températures trop basses n'assurent pas une évaporation suffisante). Au contraire, à Artigues, le vignoble est situé dans un côteau couvert de vignes et rien ne s'oppose à la propagation du signal chimique dans des secteurs, même lointains, où l'insecte est présent.

Les fortes doses seraient donc avantagées par leur distance d'attraction plus grande. La tendance observée dans l'analyse globale à une plus faible efficacité de la dose médiane $(100 \mu \mathrm{g})$ est un indice supplémentaire permettant de penser que les captures au piège sont la résultante de 2 phénomènes contraires : l'un, l'attraction, étant favorisé par les fortes doses, l'autre, la capture, par les faibles.

STOCKEL \& SUREAU (1980) ont observé également une influence de la répartition géographique de la culture hôte de l'insecte sur l'efficacité des fortes doses dans le cas de Sitotroga cerealella Oliv. Alors qu'on observe, à partir d'un certain niveau de dose, un plafonnement des captures, si l'on augmente encore, il peut y avoir cependant un nouvel accroissement des captures: les auteurs pensent que le signal arrive alors dans un autre site habité par les papillons.

Lorsque l'insecte est présent dans tout le secteur où sont transportées les molécules de la phéromone, il semble donc, pour $L$. botrana comme pour $S$. cerealella, que le nombre de captures augmente avec la dose. Pour $S$. cerealella, StOCKEL \& HUGUET (1977) ont mème dégagé une relation simple : les captures sont proportionnelles au logarithme de la dose. Il s'agit, dans les 2 cas, d'attractifs ne renfermant qu'une substance, le composé principal de la sécrétion phéromonale. Pour $L$. botrana, les seules impuretés sont des isomères soit sans action sur le mâle, soit légèrement attractifs (LALANNE CASSOU, 1979). Dans les nombreux cas où a été observée une nette baisse d'efficacité en utilisant des doses élevées, il s'agit de mélanges renfermant un pourcentage précis de substances qui seraient inhibitrices si elles étaient employées seules ou dans des proportions différentes. RoELOFS a proposé, en 1978, une hypothèse expliquant cette diminution de captures : en augmentant la quantité globale du mélange mis dans le diffuseur, on augmente celle de l'inhibiteur au-delà d'un seuil à partir duquel son action antagoniste se manifeste quelle que soit sa proportion. Ce mécanisme étant impossible dans le cas d'attractif à un seul composant, on peut comprendre facilement les différences observées entre ces 2 types de substances.

Ces essais ont tous été effectués avec plusieurs pièges dans la même parcelle. Malgré la distance de $15 \mathrm{~m}$, nous n'avons pas éliminé l'action de la concurrence, action importante signalée par WALL \& PeRRY (1981). Il est possible que les performances des appâts les moins chargés, employés seuls, soient améliorées. C'est ce que montrera une autre série d'expériences.

\section{CONCLUSION}

Pour l'avertissement, la dose employée n'a pas d'importance : toute dose comprise entre 1 et $10000 \mu \mathrm{g}$ permet de déterminer les périodes de présence des papillons. Le fait que le classement de leur efficacité varie dans une large mesure avec l'environnement explique les difficultés rencontrées lors des essais d'établissement de relations entre le nombre des captures et les populations et nous amène à rechercher l'existence d'une telle relation en utilisant de préférence les doses les plus faibles possibles.

Reçu le 17 janvier 1983. Accepté le 27 avril 1983.

\section{REMERCIEMENTS}

Nous tenons à remercier $\mathbf{M}^{\text {me }}$ Martine LETTERE, du Laboratoire des Médiateurs chimiques de l'I.N.R.A., pour la synthèse de la phéromone nécessaire à cette expérimentation ainsi que $M$. DES COINS, Directeur de ce laboratoire. Nous remercions aussi $\mathbf{M}^{\mathrm{mc}}$ BALLANDE et M. BouRSAUd qui nous ont permis d'expérimenter dans leurs vignobles ainsi que $M$. REALT, régisseur du Château Baret.

\section{RÉFÉRENCES BIBLIOGRAPHIQUES}

Baker T. C., Roelofs W. L., 1981. Initiation and termination of Oriental fruit molt male response to pheromone concentrations in the fields. Environ. Entomol., 10, 211-217.

Descoins C., Lalanne Cassou B., Samain D., 1974. Sur les attractifs sexuels synthétiques pour l'eudémis de la vigne, Lobesia botrana Schiff. (Lépidoptère Tortricidae). C.R. Acad. Sci., Paris, sér. D, 279, 907-916.

Descoins C., Samain D., Lalanne Cassou B., Gallois M., 1977. Synthèses stéréosélectives des acétoxy-1 dodécadiènes $7 \mathrm{E}, 9 \mathrm{E}$ et $7 \mathrm{E}, 9 \mathrm{Z}$ attractifs sexuels pour le mâle de l'eudémis de la vigne Lobesia (Polychrosis) botrana Den. \& Schiff., lépidoptère Tortricidae. Bull. Soc. Chim. Fr., 9-10, 941-946.

Lalanne Cassou B., 1979. Contribution à l'étude de la communication sexuelle par phéromone chez l'eudémis de la vigne Lobesia botrana Schiff. (Lepidoptera Tortricidae Olethreutinae). Thèse de Doctorat, Univ. F. et M. Curie, Paris, 247 p.
Roehrich R., Carles J. P., Darrioumerle Y., Pargade P., Lalanne Cassou B., 1976. Essais en vignoble de phéromones de synthèse pour la capture des mâles de l'eudémis Lobesia botrana Schiff. Ann. Zool. Ecol. anim., 8, 473-480.

Roehrich R., Carles J. P., Trésor C., de Vathaire M. A., 1979. -Essais de confusion sexuelle contre les tordeuses de la grappe: l'eudémis Lobesia botrana Den. et Schiff. et la cochylis Eupoecilia ambiguella Tr. Ann. Zool. Ecol. anim., 11, 659-675.

Roehrich R., Carles J. P., de Vathaire M. A., 1977. Essais complémentaires au sujet du piégeage de l'eudémis Lobesia botrana Schiff. 6-9, Les phéromones sexuelles des insectes, Montfavet, 25-27 octobre 1977. Ed. I.N.R.A., 176 p.

Roehrich R., Schmid A., 1979. Lutte intégrée en viticulture, Tordeuses de la grappe : évaluation du risque, détermination des périodes d'intervention et recherche de méthodes de lutte biologique. Proc. Int. Symp. IOBC/WPRS integrierten Pflanz. Land Forstwirtsch., Wien, 8-12 okt. 1979, 648 p., 245-253. 
Roelofs W. L., 1978. Threshold hypothesis on pheromone perception. J. chem. Ecol., 4, 685-699.

Roelofs W. L., Kochansky S., Darde R., Arn H., Rauscher S., 1973. Sex attractant of the grape vine moth Lobesia botrana. Mitt. Schweiz. entomol. Ges., 46, 71-73.

Rothschild G. H. L., 1980. Mating disruption of lepidopterouspests : current status and future prospect. Ed. Mitchell. Management of insect pests with semiochemicals. Plenum Press., New York, 414 p., 207-228.

Stockel J., 1976. Mise au point d'un type de piège sexuel I.N.R.A. pour insectes. 12-14 in: "Les phéromones sexuelles des lépidoptères, Bordeaux, 13-16 octobre 1976. Ed. I.N.R.A., 217 p.
Stockel J., Huguet J. G., 1977. Relation entre la dose de phéromone de synthèse ou le nombre de femelles vierges utilisés comme appâts et le taux de captures des mâles chez Sitotroga cerealella Oliv. (Lep. Gelechiidae). Biol. Behav., 3, 273-285.

Stockel J., Sureau F., 1980. Monitoring for the angoumois grain moth in corn. 63-73 in Ed Mitchell, Management of insect pests with semiochemicals. 514 p., Plenum Press, New York.

Wall C., Perry J. N., 1981. The behaviour of moths responding to pheromone sources in the field : a basis of discussion. 170-178, Les médiateurs chimiques, Symposium international, Versailles, 1620 nov. 1981. Ed. I.N.R.A., Publ. Les Colloques de l'I.N.R.A., 7, $414 \mathrm{p}$. 